\section{Disease overview}

\section{P.L. Pearson}

Duchenne Muscular Dystrophy. By Alan E.H. Emery. Oxford University Press: 1987. Pp.315. £35, \$55.

IN HIS foreword to this latest addition to the series Oxford Monographs in Medical Genetics, Sir John Walton expresses the opinion that single-disease monographs can never be expected to be best-sellers. I sincerely hope that this book proves to be an exception to the rule, and that it will become compulsory reading for all associated with neuromuscular disorders.

In an easily read style, Emery takes the reader through many aspects of muscular dystrophy ranging from its possible inclusion on murals of ancient Egyptian tombs to the recent contributions of recombinant DNA technology. It is possible that experts in any one field will feel Emery has given insufficient attention to his or her speciality to do it justice. However, as Emery himself states in the introduction, it is not his intention to cater solely for particular interests and the book passes rapidly from topic to topic, whetting readers' appetites without boring them, and providing an adequate bibliography.

Following a historical introduction to the disease, the author considers the clinical features and makes extensive use of his own observations, occasionally backed up by series published by other authors. Although at times the number of observations used to demonstrate a particular point is limited, this is not a major criticism because the homogeneity of a single series outweighs the numerical advantage of observations pooled from different sources. It would, however, have been more helpful if similar data had been expressed in a standard format, for example, the data on age of learning to walk expressed in the same format as that used for other age-related phenomena.

Notwithstanding such small presentation hiccups and an occasional typographical error (the most amusing being "grognosis"), the book moves smoothly from one topic to another. The text is well documented with interesting figures, and one marvels in particular at the incredible muscular enlargement and definition illustrated in a negro boy with Duchenne muscular dystrophy. The section on confirmation of the diagnosis contains pertinent data on serum levels of enzymes and clear illustrations of the muscular pathology encountered, not only in Duchenne muscular dystrophy but associated disorders also. Herein lies one of the strengths of this book, in that the reader is given enough information to understand both the clinical and genetic grounds for distinguishing Duchenne muscular dystrophy from other neuromuscular disorders.

Not unexpectedly, Emery covers aspects of the inheritance of the disease, mutation frequency, carrier detection and prenatal diagnosis clearly and succinctly. For those who still do not understand the rationale of combining risks from various sources to estimate carrier risk, this book is a must. The use of DNA markers is considered in just sufficient detail to make the technique clear to non-geneticists without getting them bogged down in minutiae.

The broad basis is kept up to the end. The final sections on genetic counselling and patient management include humane and compassionate discussions of the rights of the parents in counselling decisions and the psychological problems

\section{Poste standards}

Jack A. Lucy

Cell Fusion. Edited by Arthur E. Sowers. Plenum: 1987. Pp.540. $\$ 75$ (North America), $\$ 90$ (elsewhere).

Cell fusion is a feature of the preparation of monoclonal antibodies and, in this rather restricted guise, it is familiar enough. However, for many years the wider aspects of cell fusion and membrane fusion that underlie so many different biological phenomena have been pursued by relatively few investigators. This situation appears to be changing, judging at least by the accelerating rate at which books on the subject are appearing. Six years passed before the publication in 1978 of $\mathrm{Mem}$ brane Fusion, edited by Poste and Nicolson, was followed by a Ciba Symposium volume entitled Cell Fusion. Only three years later we have the present book with an identical title, while the proceedings of an international meeting on "Molecular Mechanisms of Membrane Fusion", held in Buffalo in April, are currently in press.

The latest volume is divided into four parts of roughly equal importance: "Fusion in Cell Membranes"; "Fusion in Model Membranes"; "New Membrane Fusion Methods"; and "Applications of Membrane Fusion". The book is an interesting and useful reference source, particularly with regard to some of the recent advances in these fields. It has been published to a high standard (although not entirely free from typographical errors), the micrographs are well reproduced and each chapter has its own bibliography in which the titles of papers are included.

The editor notes in his preface that most current work on membrane fusion is within the context of intact or modified cells, and that the book emphasizes the plasma membrane. He also comments that the material covered in the section on plasma encountered by the patients themselves.

One feels that in some respects the book has been published just a little bit too early. Rapid molecular developments in characterizing the gene are now changing our conception of what causes the highmutation frequency and giving insights into the role of the abnormal gene product to account for the differences between Becker and Duchenne muscular dystrophy. However, much of the clinical information will remain relevant and I hope that the author will be encouraged to up-date the molecular sections in the nottoo-distant future.

P. L. Pearson is a Professor in the Department of Human Genetics, Sylvius Laboratories, University of Leiden, Wassenaarseweg 72, 2333AL Leiden, The Netherlands.

membranes was selected to emphasize depth rather than breadth. Unfortunately for anyone using the book as an introduction to the field, this selectivity has apparently been responsible for the exclusion of fertilization and the associated acrosome and cortical granule reactions. Fertilization is not even mentioned in the index. The formation of multinucleate cells in inflammatory reactions by the fusion of macrophages (covered by Poste and Nicolson) is also not considered, and membrane fusion in endocytosis is given little attention.

Comparisons with Poste and Nicolson's book are rather invited because the preface refers back to it and remarks that there is an obvious need for an up-to-date monograph that integrates material on new fusion methods and their applications, reviews established material, and rationalizes and integrates the old and the new. In my view, however, the present volume does not approach the bench-mark set by Poste and Nicolson in either depth or breadth. Although it contains eleven more chapters, it is about 40 per cent shorter and several of the contributions are straightforward accounts of new work which would be much more appropriately published in a regular research journal.

The new book is also much less catholic in its representation of international research. Only two of the 26 chapters are from laboratories outside the United States, compared with half of those in Poste and Nicolson's volume. This is particularly noticeable in relation to electrofusion. Many significant papers have been published on the fundamental importance and potential applications of electrofusion by laboratories in Germany and France, but there are no contributions from them among the five chapters on this topic.

Jack A. Lucy is Professor of Biochemistry at the Royal Free Hospital School of Medicine, University of London, Rowland Hill Street, London NW3 $2 P F, U K$ 